University of Nebraska - Lincoln

DigitalCommons@University of Nebraska - Lincoln

Faculty Publications: Materials Research

Science and Engineering Center

Materials Research Science and Engineering

Center

May 2003

\title{
Atomic, electronic, and magnetic properties of magnetic tunnel junctions
}

I.I. Oleynik

University of South Florida, Tampa

Evgeny Y. Tsymbal

University of Nebraska-Lincoln, tsymbal@unl.edu

Follow this and additional works at: https://digitalcommons.unl.edu/mrsecfacpubs

Part of the Materials Science and Engineering Commons

Oleynik, I.I. and Tsymbal, Evgeny Y., "Atomic, electronic, and magnetic properties of magnetic tunnel junctions" (2003). Faculty Publications: Materials Research Science and Engineering Center. 40. https://digitalcommons.unl.edu/mrsecfacpubs/40

This Article is brought to you for free and open access by the Materials Research Science and Engineering Center at DigitalCommons@University of Nebraska - Lincoln. It has been accepted for inclusion in Faculty Publications: Materials Research Science and Engineering Center by an authorized administrator of DigitalCommons@University of Nebraska - Lincoln. 


\title{
Atomic, electronic, and magnetic properties of magnetic tunnel junctions
}

\author{
I. I. Oleynik ${ }^{a}$ \\ Department of Physics, University of South Florida, Tampa, Florida 33620-5700 \\ E. Yu. Tsymbal \\ Department of Physics and Astronomy, University of Nebraska-Lincoln, Lincoln, Nebraska 68588
}

(Presented on 12 November 2002)

\begin{abstract}
We report results of first-principles density-functional studies of the atomic and electronic structure of $\mathrm{Co} / \mathrm{Al}_{2} \mathrm{O}_{3} / \mathrm{Co}$ and $\mathrm{Co} / \mathrm{SrTiO}_{3} / \mathrm{Co}$ magnetic tunnel junctions (MTJs). The atomic structure has been studied for different interface terminations and the interfacial energetics was quantified based on the work of separation. Based on energetics of cohesion, the O-terminated and $\mathrm{TiO}_{2}$-terminated interfaces were identified as the most stable structures for $\mathrm{Co} / \mathrm{Al}_{2} \mathrm{O}_{3} / \mathrm{Co}$ and $\mathrm{Co} / \mathrm{SrTiO}_{3} / \mathrm{Co}$ MTJs, respectively. The electronic structure was analyzed using local densities of states. We found that the electronic structure of the O-terminated $\mathrm{Co} / \mathrm{Al}_{2} \mathrm{O}_{3} / \mathrm{Co}$ tunnel junction exhibits negative spin polarization at the Fermi energy within the first few monolayers of alumina but it eventually becomes positive for distances beyond $10 \AA$. Calculated electronic structure of the $\mathrm{TiO}_{2}$-terminated $\mathrm{Co} / \mathrm{SrTiO}_{3} / \mathrm{Co} \mathrm{MTJ}$ shows an exchange coupling between the interface $\mathrm{Co}$ and $\mathrm{Ti}$ atoms mediated by oxygen. This coupling induces a magnetic moment of $0.25 \mu_{B}$ on the interface Ti atom, which is aligned antiparallel to the magnetic moment of the Co layer. This is a possible cause of inversion of the spin polarization of tunneling across the $\mathrm{SrTiO}_{3}$ barrier that was found in recent experiments. (C) 2003 American Institute of Physics. [DOI: 10.1063/1.1558631]
\end{abstract}

Recent experiments have suggested that the spin degrees of freedom of the electron can be utilized to enhance the performance of existing semiconductor devices. ${ }^{1}$ Magnetic tunnel junctions (MTJs) consisting of spin-polarized ferromagnetic electrodes sandwiching an insulating barrier are such promising candidates of spintronic devices for applications as magnetic random access memory (MRAM). ${ }^{2,3}$ The underlying principle of functioning of these MRAMs exploits the physics of spin-dependent tunneling. The tunneling magnetoresistance, i.e., the relative difference in the resistance between parallel and antiparallel magnetizations of the electrodes is related to the spin polarization (SP) of the tunneling conductance. ${ }^{4}$ It was believed that the magnitude of the TMR is determined solely by the spin polarization of the density of states of bulk ferromagnets at the Fermi energy. ${ }^{5}$ However, recent experimental and theoretical investigations show that the SP of the conductance in MTJs is not an intrinsic property of the ferromagnet alone but depends on the electronic properties of the insulator and the ferromagnetinsulator interface.

This idea was investigated in greater detail in a series of recent experiments, ${ }^{6}$ where a positive SP was found for the $\mathrm{Al}_{2} \mathrm{O}_{3}$ barrier, but a negative $\mathrm{SP}$ was measured for the $\mathrm{SrTiO}_{3}$ barrier for the tunneling current from the Co electrode. It was also shown that this negative SP is actually controlled by a few monolayers of $\mathrm{SrTiO}_{3}$ adjacent to the interface with the Co layer. ${ }^{6}$ This comparative study highlights the importance of several factors controlling spindependent tunneling, such as the chemical composition of the barrier and the atomic structure and bonding at the

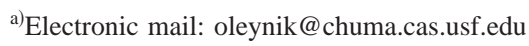

metal-oxide interfaces. In the present article, we report results of first-principle density-functional calculations of the atomic and electronic structure of $\mathrm{Co} / \mathrm{Al}_{2} \mathrm{O}_{3} / \mathrm{Co}$ and $\mathrm{Co} / \mathrm{SrTiO}_{3} / \mathrm{Co}$ MTJs aimed at providing the theoretical input for interpreting the results of the experimental studies of spin-dependent tunneling.

The calculations are based on the spin-polarized densityfunctional theory within the generalized gradient approximation in a plane-wave pseudopotential implementation. Due to the lack of detailed experimental information for the atomic structure of the MTJs, we constructed models which incorporate the growth conditions. Since $\mathrm{Co} / \mathrm{Al}_{2} \mathrm{O}_{3} / \mathrm{Co}$ MTJs are produced by depositing a few tens of monolayers of aluminum film on top of the crystalline face-centered-cubic (fcc) Co layer, followed by thermal or plasma assisted oxidation, ${ }^{7}$ we have considered crystalline $\alpha-\mathrm{Al}_{2} \mathrm{O}_{3}$ with the [0001] orientation on top of fcc (111) Co. For the case of a $\mathrm{Co} / \mathrm{SrTiO}_{3} / \mathrm{Co} \mathrm{MTJ}$, a fcc Co layer is grown on top of the strontium titanate (001) surface, ${ }^{6}$ therefore, it is reasonable to assume that the Co (100) layer is expanded to accommodate the lattice mismatch between $\mathrm{Co}$ and $\mathrm{SrTiO}_{3}$. The relaxed atomic structure of the MTJ is determined by optimizing all the internal coordinates of the atoms as well as the height of the unit cell under constraint of the fixed lateral cell dimensions.

We have considered different interface terminations of the MTJs and, in order to determine the most stable structure among different terminations, we calculated the work of separation that quantifies the energetics of adhesion between the ferromagnet and insulator. ${ }^{8}$ For the case of a $\mathrm{Co} / \mathrm{Al}_{2} \mathrm{O}_{3} / \mathrm{Co}$ MTJ, the O-terminated interface is the most stable one with the work of separation of $6.35 \mathrm{~J} / \mathrm{m}^{2}$ [see Fig. 1(a)] whereas the most stable $\mathrm{TiO}_{2}$-terminated interface in a 


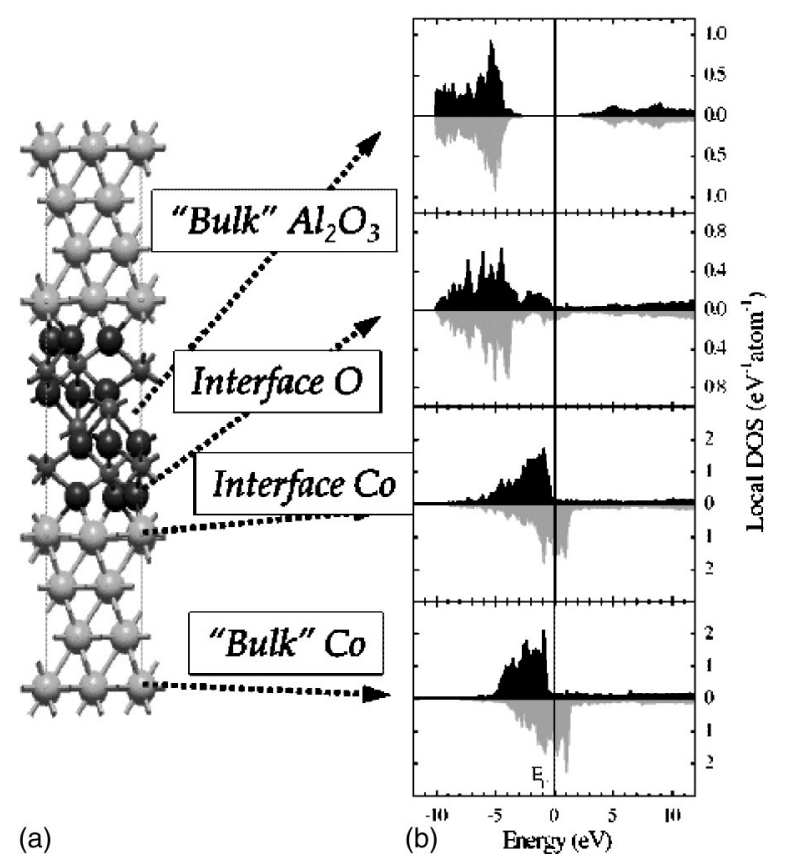

FIG. 1. Atomic (a) and electronic (b) structures of O-terminated $\mathrm{Co} / \mathrm{Al}_{2} \mathrm{O}_{3} / \mathrm{Co} \mathrm{MTJ}$.

$\mathrm{Co} / \mathrm{SrTiO}_{3} / \mathrm{Co} \mathrm{MTJ}$ has a maximum work of separation of $3.39 \mathrm{~J} / \mathrm{m}^{2}$ [see Fig. 2(a)].

The resulting spin- and layer-dependent density of states (DOS) for the O-terminated interface are shown in Fig. 1(b). The top panel displays the local DOS (LDOS) in the middle of the oxide $\alpha-\mathrm{Al}_{2} \mathrm{O}_{3}$. We found that these LDOS are very similar to those obtained for the bulk $\alpha-\mathrm{Al}_{2} \mathrm{O}_{3}$. In the bulk $\alpha-\mathrm{Al}_{2} \mathrm{O}_{3}$ the valence and the conduction bands are separated by a band gap which is, according to our results, equal to 6.2

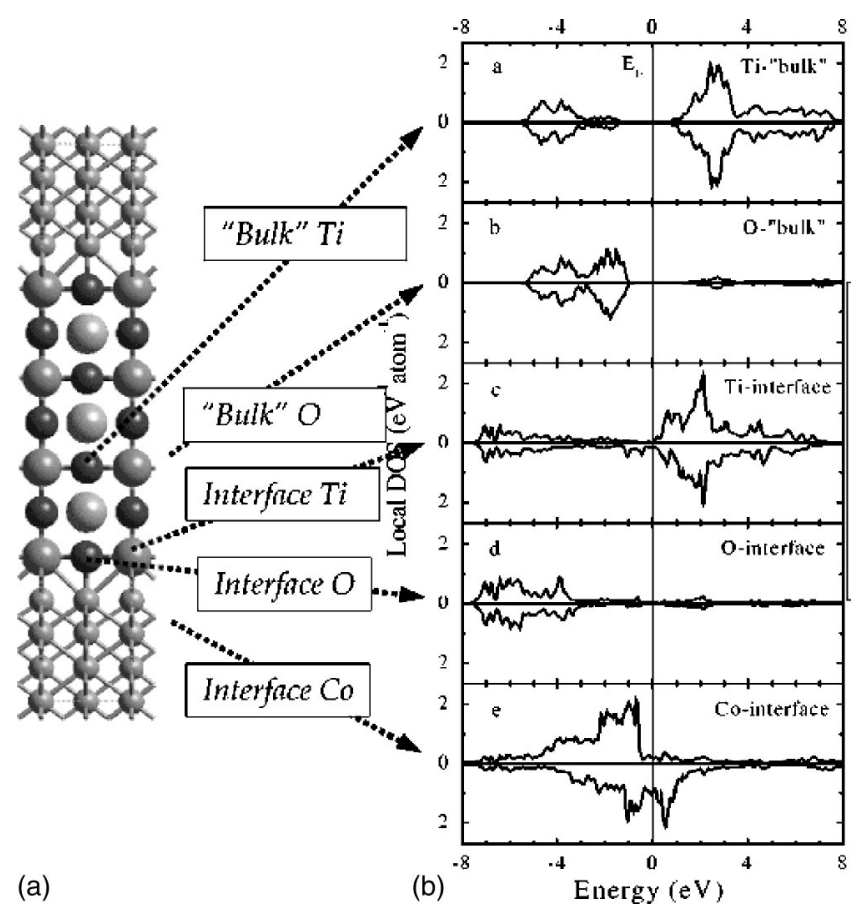

FIG. 2. Atomic (a) and electronic (b) structures of O-terminated $\mathrm{Co} / \mathrm{SrTiO}_{3} / \mathrm{Co} \mathrm{MTJ}$.
$\mathrm{eV}$ at the $\Gamma$-point. This value is less than the experimental band gap of $8.8 \mathrm{eV}$ as a result of using the local density approximation (LDA). In the presence of the interface with the Co metal, the LDOS within the band gap of alumina is not exactly zero showing the presence of electronic states of the Co metal that propagate into the insulator barrier, decaying exponentially with the distance in oxide layer. These metal-induced states are spin polarized and are responsible for the spin-dependent tunneling. ${ }^{4}$

The electronic structure of the $\mathrm{Co} / \mathrm{SrTiO}_{3} / \mathrm{Co}$ MTJ is shown in Fig. 2(b). In the bulk $\mathrm{SrTiO}_{3}$, the valence and conduction bands are separated by a band gap, which is, according to our results, equal to $2.1 \mathrm{eV}$ at the $\Gamma$-point. Again, this value is less than the experimental band gap of $3.3 \mathrm{eV}$ as a result of using the LDA. The electronic interactions at the $\mathrm{Co} / \mathrm{TiO}_{2}$ interface are dominated by strong covalent bonding between the $2 p$ orbitals of oxygen and the $3 d$ orbitals of cobalt. This strong $\mathrm{Co}-\mathrm{O}$ covalent bonding pushes down the bonding states at the interface $\mathrm{O}$ atom, which appear in the region of $6-7 \mathrm{eV}$ below the Fermi energy [see Fig. 2(b)]. Interestingly, there is almost no induced exchange splitting of these states (at least within the $0.1 \mathrm{eV}$ energy scale), which is different to what we found for the O-terminated $\mathrm{Co} / \mathrm{Al}_{2} \mathrm{O}_{3}$ interface, see Fig. 1(b).

Similar to the $\mathrm{Co} / \mathrm{Al}_{2} \mathrm{O}_{3}$ interface, one can see some evidence of the exchange splitting of these states in the $2 \mathrm{eV}$ interval below the Fermi level. However, contrary to the cobalt-alumina case, the density of these exchange-split antibonding states is much less and, in addition, they lie below the Fermi energy (i.e., are occupied) for both the majority and minority spins. This results in a negligible magnetic moment on the interface $\mathrm{O}$ atoms. The interface-chemical interactions substantially affect the LDOS of the interface Ti layer, as can be seen from Fig. 2(b). Although we find that there is no direct bonding between the interfacial $\mathrm{Ti}$ and $\mathrm{Co}$ atoms, the indirect coupling mediated by the $\mathrm{O}$ atoms has a sizable affect on the electronic structure of Ti at the interface. The most important consequence of this coupling is the formation of the exchange-split states in the interval of energies $[-1,+1] \mathrm{eV}$. As is seen from the bottom panel in Fig. 2(b), the minority states (the two peaks at $-1 \mathrm{eV}$ and $-0.5 \mathrm{eV}$ ) are occupied, whereas the majority states lie above the Fermi energy and are overlapped with the states in the conduction band (the two peaks at $+0.5 \mathrm{eV}$ and $+1 \mathrm{eV}$ ). This leads to an induced magnetic moment of $0.25 \mu_{B}$ on the interfacial Ti atoms, which is aligned antiparallel to the magnetic moment of the Co layer.

Although we have not calculated the tunneling conductance yet, we can make some conclusions about the mechanism of spin-dependent tunneling based on the LDOS considerations. Obviously, the LDOS of the left- and right-hand side electrodes explicitly enters the expression for the tunneling current and, in the limit of large barrier thicknesses, the tunneling current is simply proportional to the products of the LDOS at the Fermi level for the left- and right-hand side electrodes in the middle of the tunneling barrier. This is relationship is analogous to the famous Tersoff-Hamman principle $^{9}$ in the theory of scanning tunneling microscopy (STM) that states the proportionality of the STM current to 


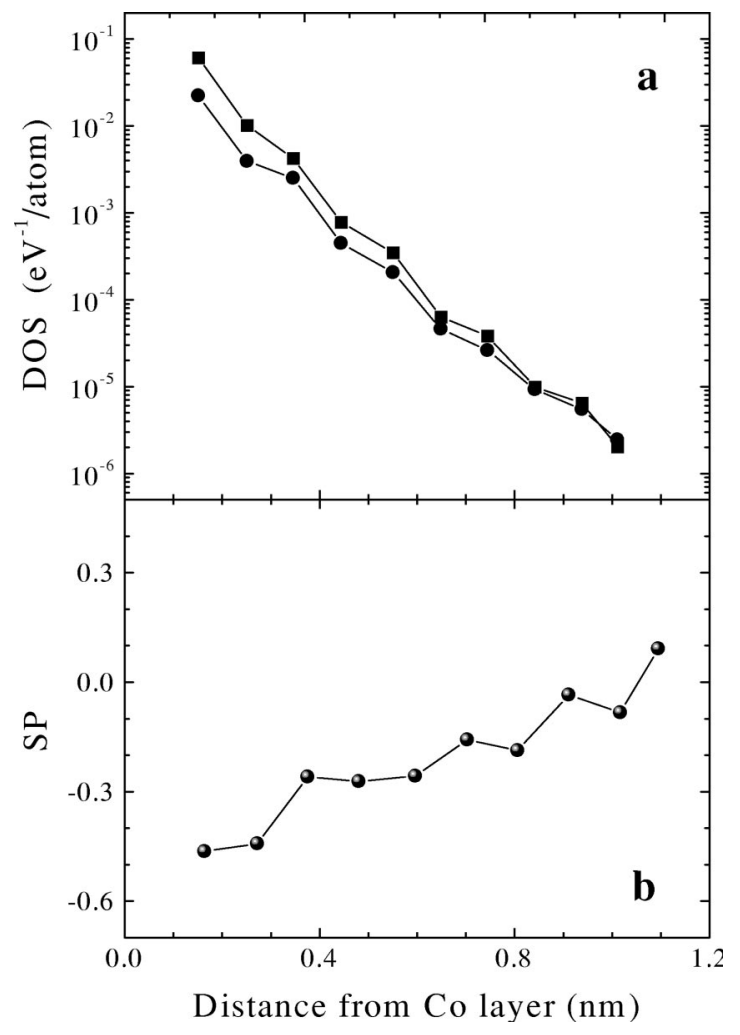

FIG. 3. LDOS for minority (squares) and majority (circles) spin electrons (a), and SP at the Fermi energy as a function of distance from interface toward insulator for $\mathrm{Co} / \mathrm{Al}_{2} \mathrm{O}_{3} / \mathrm{Co} \mathrm{MTJ}$ (b).

the LDOS of the sample at the position of the STM tip. In case of O-terminated $\mathrm{Co} / \mathrm{Al}_{2} \mathrm{O}_{3} / \mathrm{Co}$, Fig. 3(a) illustrates the distribution of the LDOS at the Fermi energy for the majority and minority spin electrons inside the isulator, i.e., as a function of the distance from the interfacial Co layer to the middle of the tunneling barrier. Evidently, the LDOS decreases exponentially with distance. Although the minority DOS is larger at distances near the interface, it decays more rapidly as compared to the majority LDOS. Eventually, the majority LDOS starts to dominate the minority LDOS resulting in the positive SP. This can be seen from Fig. 3(a), which shows the SP of the DOS at the Fermi level as a function of distance from the interface Co layer. The SP is defined by $\left(D_{\uparrow}-D_{\downarrow}\right) /\left(D_{\uparrow}+D_{\downarrow}\right)$, where $D_{\uparrow}$ and $D_{\downarrow}$ are the LDOS for the majority and minority spins, respectively. It is clear from Fig. 3(b), the layer-averaged SP increases gradually without showing a tendency for saturation. Therefore, the LDOS of the O-terminated $\mathrm{Co} / \mathrm{Al}_{2} \mathrm{O}_{3} / \mathrm{Co}$ MTJ becomes $100 \%$ positive spin-polarized in the limit of large insulator thickness. This reflects the fact that the $d$ states of cobalt decay faster in the insulator barrier that the $s-p$ states.

In case of the $\mathrm{Co} / \mathrm{SrTiO}_{3} / \mathrm{Co}$ MTJ, the induced antiparallel-aligned magnetic moment on the interfacial $\mathrm{Ti}$ atoms might have an important consequence for spindependent tunneling across $\mathrm{SrTiO}_{3}$ compared to $\mathrm{Al}_{2} \mathrm{O}_{3}$. As is known from experiment, ${ }^{10}$ by modifying the electronic structure of the interfacial layers, it is possible to change the spin polarization of the conductance. The presence of the antiparallel-aligned magnetic moment of the Ti atoms may cause the same effect as changing the direction of the magnetic moment of the ferromagnetic layer at the interface to the opposite. This may result in the inversion of the SP, which was found in experiment. ${ }^{6}$ The negative SP is also indicated by the fact that the Ti LDOS at the Fermi energy is larger by a factor of 3 for the minority spins than for the majority spins [see Fig. 2(b)].

In conclusion, we have studied the atomic and electronic structure of the $\mathrm{Co} / \mathrm{Al}_{2} \mathrm{O}_{3} / \mathrm{Co}$ and $\mathrm{Co} / \mathrm{SrTiO}_{3} / \mathrm{Co}$ MTJs using first-principles density-functional theory. The most stable structures are the O-terminated interface in the $\mathrm{Co} / \mathrm{Al}_{2} \mathrm{O}_{3} / \mathrm{Co}$ MTJ and the $\mathrm{TiO}_{2}$-terminated interface in the case of the $\mathrm{Co} / \mathrm{SrTiO}_{3} / \mathrm{Co} \mathrm{MTJ}$. We found that the electronic structure of the O-terminated $\mathrm{Co} / \mathrm{Al}_{2} \mathrm{O}_{3} / \mathrm{Co}$ MTJ exhibits negative SP at the Fermi energy within the first few monolayers of alumina but it eventually becomes positive for distances beyond $10 \AA$. The $\mathrm{Co} / \mathrm{SrTiO}_{3} / \mathrm{Co}$ MTJ shows an exchange coupling between the interface $\mathrm{Co}$ and $\mathrm{Ti}$ atoms mediated by oxygen, which results in an induced magnetic moment of $0.25 \mu_{B}$. This magnetic moment is aligned antiparallel to the magnetic moment of the Co layer, which may lead to a negative SP of tunneling across the $\mathrm{SrTiO}_{3}$ barrier from the Co electrode. The results of our calculations provide necessary information for more detailed theoretical investigation of the spindependent transport in MTJs. Our results illustrate the important fact that spin-polarized tunneling in MTJs is not determined entirely by bulk DOS of ferromagnet electrodes, but is also very sensitive to the nature of the insulating tunneling barrier, as well as the atomic structure and bonding at ferromagnet/insulator interface. This conclusion is in resonance with recent theoretical ${ }^{11}$ and experimental ${ }^{12}$ works that stress the importance of interface effects in spin-polarized tunneling.

${ }^{1}$ S. A. Wolf, D. D. Awschalom, R. A. Buhrman, J. M. Daughton, S. von Molnar, M. L. Roukes, A. Y. Chtchelkanova, and D. M. Treger, Science 294, 488 (2001)

${ }^{2}$ J. S. Moodera, J. Nassar, and G. Mathon, Annu. Rev. Mater. Sci. 29, 381 (1999).

${ }^{3}$ J. M. Slaughter, E. Y. Chen, R. Whig, B. N. Engel, J. Janesky, and S. Tehrani, JOM-e, 52, 6 (2000).

${ }^{4}$ R. Meservey and P. M. Tedrow, Phys. Rep. 238, 173 (1994).

${ }^{5}$ M. Julliere, Phys. Lett. A 54, 225 (1975).

${ }^{6}$ J. M. De Teresa, A. Barthelemy, A. Fert, J. P. Contour, R. Lyonnet, F. Montaigne, P. Seneor, and A. Vaures, Phys. Rev. Lett. 82, 4288 (1999).

${ }^{7}$ D. J. Monsma and S. S. P. Parkin, Appl. Phys. Lett. 77, 883 (2000).

${ }^{8}$ M. W. Finnis, J. Phys.: Condens. Matter 8, 5811 (1996).

${ }^{9}$ J. Tersoff and D. R. Hamman, Phys. Rev. B 31, 805 (1985).

${ }^{10}$ P. LeClair, J. T. Kohlhepp, H. J. M. Swagten, and W. J. M. de Jonge, Phys. Rev. Lett. 86, 1066 (2001).

${ }^{11}$ C. Uiberacker and P. M. Levy, Phys. Rev. B 64, 193404 (2001).

${ }^{12} \mathrm{M}$. G. Muenzenberg and J. S. Moodera (unpublished). 